\title{
THE RADIUS OF UNIVALENCE OF CERTAIN ANALYTIC FUNCTIONS
}

\author{
THOMAS H. MACGREGOR
}

1. Introduction. Suppose that $f(z)=z+a_{2} z^{2}+\cdots$ is analytic for $|z|<1$. If $\operatorname{Re}\{f(z) / z\}>0$ for $|z|<1$ then $f(z)$ is univalent in $|z|$ $<\sqrt{2}-1[5$, Theorem $3 ; 7]$. The function $f(z)=\left(z+z^{2}\right) /(1-z)$ satisfies the hypotheses but is univalent in no circle $|z|\langle r$ for $r\rangle \sqrt{2}-1$ since its derivative vanishes at $z=\sqrt{2}-1$.

In this paper we generalize the above theorem for functions whose power series begins $f(z)=z+a_{n+1} z^{n+1}+\cdots$. The estimate used to obtain this result is further used to find the radius of convexity for functions $f(z)=z+a_{n+1} z^{n+1}+\cdots$ which are analytic and satisfy $\operatorname{Re} f^{\prime}(z)>0$ for $|z|<1$. For $n=1$ this theorem is not new [5, Theorem $2 ; 10$, p. 284]. The condition $\operatorname{Re} f^{\prime}(z)>0$ is known to be sufficient for the univalency of $f(z)$ in $|z|<1[1$, p. 18].

We consider the problem of finding the radius of univalence for functions $f(z)=z+a_{2} z^{2}+\cdots$ which are analytic and satisfy $\operatorname{Re}\{f(z) / g(z)\}>0$ for $|z|<1$, where $g(z)=z+b_{2} z^{2}+\cdots$ is analytic and univalent for $|z|<1$. In the case that $g(z)$ is either starlike or convex this problem is solved. We take particular advantage of the condition $\operatorname{Re}\left\{z f^{\prime}(z) / f(z)\right\}>0$ for $|z|<r$, which is necessary and sufficient for $f(z)$ to be univalent and starlike in $|z|<r$ [8, p. 105, problem 109]. For arbitrary univalent functions $g(z)$ we only obtain an estimate for the radius of univalence for $f(z)$.

2. Lemma 1. Suppose that $h(z)=1+c_{n} z^{n}+\cdots$ is analytic and satisfies $\operatorname{Re} h(z)>0$ for $|z|<1$. Then

$$
\left|\frac{h^{\prime}(z)}{h(z)}\right| \leqq \frac{2 n|z|^{n-1}}{1-|z|^{2 n}} .
$$

Proof. Let $k(z)=(1-h(z)) /(1+h(z))=d_{n} z^{n}+\cdots$. Then $k(z)$ is analytic for $|z|<1$ and $|k(z)|<1$. Thus, $k(z)=z^{n} \phi(z)$ where $\phi(z)$ is analytic for $|z|<1$ and $|\phi(z)| \leqq 1$. For such functions we have

$$
\left|\phi^{\prime}(z)\right| \leqq \frac{1-|\phi(z)|^{2}}{1-|z|^{2}}
$$

[2, p. 18].

Expressing $h(z)$ and $h^{\prime}(z)$ in terms of $\phi(z)$ gives

Received by the editors January 11, 1962 and, in revised form, March 12, 1962. 


$$
\begin{aligned}
\frac{h^{\prime}(z)}{h(z)} & =-2 z^{n-1} \frac{z \phi^{\prime}(z)+n \phi(z)}{1-z^{2 n} \phi^{2}(z)} \\
\left|\frac{h^{\prime}(z)}{h(z)}\right| & \leqq 2|z|^{n-1} \frac{|z|\left|\phi^{\prime}(z)\right|+n|\phi(z)|}{1-|z|^{2 n}|\phi(z)|^{2}} .
\end{aligned}
$$

Using (1) we obtain

$$
\left|\frac{h^{\prime}(z)}{h(z)}\right| \leqq \frac{2|z|^{n-1}}{1-|z|^{2}} \frac{|z|\left(1-|\phi(z)|^{2}\right)+n\left(1-|z|^{2}\right)|\phi(z)|}{1-|z|^{2 n}|\phi(z)|^{2}} .
$$

${ }^{1}$ To prove the lemma it is sufficient to show that for $|z|=r$, $0<r<1$,

$$
\frac{r\left(1-|\phi(z)|^{2}\right)+n\left(1-r^{2}\right)|\phi(z)|}{1-r^{2 n}|\phi(z)|^{2}} \leqq \frac{n\left(1-r^{2}\right)}{1-r^{2 n}} .
$$

Letting $x=|\phi(z)|$ this is equivalent to $(1-x) F_{n}(x) \geqq 0$ for $0 \leqq x \leqq 1$, where

$$
\begin{aligned}
F_{n}(x) & =a-b x, \quad a=n\left(1-r^{2}\right)-r\left(1-r^{2 n}\right)>\left(1-r^{2}\right)(n-n r)>0, \\
b & =r\left(1-r^{2 n}\right)-n r^{2 n}\left(1-r^{2}\right) \\
& =r\left(1-r^{2}\right)\left(1+r^{2}+r^{4}+\cdots+r^{2 n-2}-n r^{2 n-1}\right) \\
& =r\left(1-r^{2}\right)\left\{\left(1-r^{2 n-1}\right)+\left(r^{2}-r^{2 n-1}\right)+\cdots+\left(r^{2 n-2}-r^{2 n-1}\right)\right\} \\
& >0 .
\end{aligned}
$$

Since $F_{n}(x) \geqq F_{n}(1)$ we can prove $F_{n}(x) \geqq 0$ by showing that $F_{n+1}(1) \geqq F_{n}(1)$ and $F_{1}(1) \geqq 0$.

$$
\begin{aligned}
& F_{n+1}(1)-F_{n}(1) \\
& \quad=\left(1-r^{2}\right)\left(1-r^{2 n+1}-r^{2 n+1}+r^{2 n+2}-n r^{2 n}+n r^{2 n+2}\right) \\
& \quad=\left(1-r^{2}\right)(1-r)\left\{1+r+r^{2}+\cdots+r^{2 n}-r^{2 n+1}-n r^{2 n}-n r^{2 n+1}\right\} \\
& \quad>0 .
\end{aligned}
$$

This inequality follows since the negative terms in the brackets can be expressed as $2 n+1$ terms each of which is numerically less than a corresponding positive term.

Finally, $F_{1}(1)=(1+r)(1-r)^{3}>0$.

One can show that the equality holds in the lemma only for the functions $h(z)=\left(1-\epsilon z^{n}\right) /\left(1+\epsilon z^{n}\right)$ where $|\epsilon|=1$ and for appropriate values of $z$.

THEOREM 1. Suppose that $f(z)=z+a_{n+1} z^{n+1}+\cdots$ is analytic and

1 I would like to thank the referee of this paper for simplifying my argument for the remaining part of the proof. 
satisfies $\operatorname{Re}\{f(z) / z\}>0$ for $|z|<1$. Then $f(z)$ is univalent and starlike in $|z|<\left(\left(n^{2}+1\right)^{1 / 2}-n\right)^{1 / n}$.

Proof. Since $\operatorname{Re}\{f(z) / z\}>0$ we can infer that $f(z)$ cannot vanish in $|z|<1$ except for a simple zero at $z=0$. Let

$$
h(z)=\frac{f(z)}{z}=1+a_{n+1} z^{n}+\cdots, \operatorname{Re} h(z)>0 \quad \text { for }|z|<1 .
$$

From Lemma 1 we have

$$
\left|\frac{z h^{\prime}(z)}{h(z)}\right| \leqq \frac{2 n|z|^{n}}{1-|z|^{2 n}} .
$$

Also

$$
\frac{z f^{\prime}(z)}{f(z)}=1+\frac{z h^{\prime}(z)}{h(z)} .
$$

Therefore, $f(z)$ will be univalent and starlike if $\left|z h^{\prime}(z) / h(z)\right|<1$. From the above estimate this is satisfied if $\left(2 n|z|^{n}\right) /\left(1-|z|^{2 n}\right)<1$, i.e., for $|z|<\left(\left(n^{2}+1\right)^{1 / 2}-n\right)^{1 / n}$.

The function $f(z)=\left(z+z^{n+1}\right) /\left(1-z^{n}\right)=z+2 z^{n+1}+\cdots$ satisfies

$$
\operatorname{Re}\left\{\frac{f(z)}{z}\right\}>0 \quad \text { for }|z|<1
$$

but is not univalent in $|z|<r$ for $r>r_{n}=\left(\left(n^{2}+1\right)^{1 / 2}-n\right)^{1 / n}$ since $f^{\prime}\left(r_{n} e^{i(x / n)}\right)=0$.

TheOREM 2. Suppose that $f(z)=z+a_{n+1} z^{n+1}+\cdots$ is analytic and satisfies $\operatorname{Re} f^{\prime}(z)>0$ for $|z|<1$. Then $f(z)$ is convex in $|z|<\left(\left(n^{2}+1\right)^{1 / 2}\right.$ $-n)^{1 / n}$.

Proof. We can apply Lemma 1 to $f^{\prime}(z)=1+(n+1) a_{n+1} z^{n}+\ldots$ since $\operatorname{Re} f^{\prime}(z)>0$. This gives $\left|f^{\prime \prime}(z) / f^{\prime}(z)\right| \leqq 2 n|z|^{n-1} / 1-|z|^{2 n}$. The condition $\operatorname{Re}\left\{\left(z f^{\prime \prime}(z) / f^{\prime}(z)\right)+1\right\}>0$ for $|z|<r$ is necessary and sufficient for $f(z)$ to map $|z|<r$ onto a convex domain [8, problem 108 , p. 105]. This condition is satisfied if $\left|z f^{\prime \prime}(z) / f^{\prime}(z)\right|<1$. From the above estimate we can deduce that $f(z)$ is convex if $\left(2 n|z|^{n}\right) /\left(1-|z|^{2 n}\right)<1$. This inequality is equivalent to $|z|<\left(\left(n^{2}+1\right)^{1 / 2}-n\right)^{1 / n}$.

The function

$$
f(z)=\int_{0}^{z} \frac{1+\sigma^{n}}{1-\sigma^{n}} d \sigma=z+\frac{2}{n+1} z^{n+1}+\cdots
$$

is an extremal function for Theorem 2 . 
3. Theorem 3. Suppose that $f(z)=z+a_{2} z^{2}+\cdots$ and $g(z)=z$ $+b_{2} z^{2}+\cdots$ are analytic for $|z|<1$ and $g(z)$ is univalent and starlike for $|z|<1$. If $\operatorname{Re}\{f(z) / g(z)\}>0$ for $|z|<1$ then $f(z)$ is univalent and starlike in $|z|<2-\sqrt{3}$.

Proof. The hypotheses imply that $f(z)$ and $g(z)$ do not vanish in $|z|<1$ except for the simple zero at $z=0$. Let

$$
h(z)=\frac{f(z)}{g(z)}=1+c_{1} z+\cdots, \operatorname{Re} h(z)>0 \quad \text { for }|z|<1 .
$$

Applying Lemma 1 to $h(z)$ for $n=1$ gives $\left|z h^{\prime}(z) / h(z)\right|$ $\leqq 2|z| /\left(1-|z|^{2}\right)$. Since $g(z)$ is starlike $\operatorname{Re}\left\{z g^{\prime}(z) / g(z)\right\}>0$ for $|z|<1$. Thus $\operatorname{Re}\left\{z g^{\prime}(z) / g(z)\right\} \geqq(1-|z|) /(1+|z|)$ [8, problem 287 , p. 140].

$$
\begin{aligned}
\frac{z f^{\prime}(z)}{f(z)} & =\frac{z g^{\prime}(z)}{g(z)}+\frac{z h^{\prime}(z)}{h(z)} \\
\operatorname{Re}\left\{\frac{z f^{\prime}(z)}{f(z)}\right\} & \geqq \operatorname{Re}\left\{\frac{z g^{\prime}(z)}{g(z)}\right\}-\left|\frac{z h^{\prime}(z)}{h(z)}\right| \geqq \frac{1-|z|}{1+|z|}-\frac{2|z|}{1-|z|^{2}} \\
& =\frac{1-4|z|+|z|^{2}}{1-|z|^{2}} .
\end{aligned}
$$

Thus, $\operatorname{Re}\left\{z f^{\prime}(z) / f(z)\right\}>0$ if $1-4|z|+|z|^{2}>0$. The last inequality is satisfied for $|z|<2-\sqrt{3}$. Therefore $f(z)$ is univalent in $|z|<2-\sqrt{3}$ and maps that circle onto a starlike domain.

The function $f(z)=\left(z+z^{2}\right) /(1-z)^{3}$ satisfies the hypotheses of Theorem 3 where $g(z)=z /(1-z)^{2}$ and $h(z)=(1+z) /(1-z)$. The derivative of this function vanishes at $z=\sqrt{3}-2$. Thus, it is univalent in no circle $|z|<r$ with $r>2-\sqrt{3}$.

For a part of the next theorem we need a sharpening of Lemma 1 for $n=1$. This result is known but we give a short proof of it here.

Lemma 2. Suppose that $h(z)=1+c_{1} z+\cdots$ is analytic and satisfies $\operatorname{Re} h(z)>0$ for $|z|<1$. Then $\left|h^{\prime}(z)\right| \leqq 2 \operatorname{Re} h(z) /\left(1-|z|^{2}\right)$.

Proof. ${ }^{2}$ Let $\phi(z)=(1-h(z)) /(1+h(z)),|\phi(z)|<1$ for $|z|<1$. Using

$$
\left|\phi^{\prime}(z)\right| \leqq \frac{1-|\phi(z)|^{2}}{1-|z|^{2}}
$$

gives

2 The author thanks the referee for indicating that the proof of this lemma can be obtained so readily from the estimate (1). 


$$
\left|h^{\prime}(z)\right| \leqq \frac{|1+h(z)|^{2}-|1-h(z)|^{2}}{2\left(1-|z|^{2}\right)} .
$$

The lemma follows by noting that $|1+h(z)|^{2}-|1-h(z)|^{2}=4 \operatorname{Re} h(z)$.

THEOREM 4. Suppose that $f(z)=z+a_{2} z^{2}+\cdots$ and $g(z)=z$ $+b_{2} z^{2}+\cdots$ are analytic for $|z|<1$ and $g(z)$ is univalent and convex for $|z|<1$. If $\operatorname{Re}\{f(z) / g(z)\}>0$ for $|z|<1$ then $\operatorname{Re}\left\{f^{\prime}(z) / g^{\prime}(z)\right\}>0$ for $|z|<\frac{1}{3}$. Also, $f(z)$ is univalent and starlike for $|z|<\frac{1}{3}$.

PRoof. The hypotheses imply that $f(z), g(z)$ and $g^{\prime}(z)$ do not vanish in $|z|<1$ except for the simple zeros of $f(z)$ and $g(z)$ at $z=0$. Let $h(z)=f(z) / g(z)=1+c_{1} z+\cdots, \operatorname{Re} h(z)>0$ for $|z|<1$.

Applying Lemma 2 to $h(z)$ gives $\left|h^{\prime}(z)\right| \leqq 2 \operatorname{Re} h(z) /\left(1-|z|^{2}\right)$. Since $g(z)$ is univalent and convex for $|z|<1$ we have $\operatorname{Re}\left\{z g^{\prime}(z) / g(z)\right\}$ $>\frac{1}{2}$ for $|z|<1$ and consequently $\operatorname{Re}\left\{z g^{\prime}(z) / g(z)\right\} \geqq(1+|z|)^{-1}[6 ; 9]$. This implies $\left|g(z) / g^{\prime}(z)\right| \leqq|z|(1+|z|)$.

$$
\begin{aligned}
\frac{f^{\prime}(z)}{g^{\prime}(z)} & =h(z)+\frac{g(z)}{g^{\prime}(z)} h^{\prime}(z) \\
\operatorname{Re}\left\{\frac{f^{\prime}(z)}{g^{\prime}(z)}\right\} & \geqq \operatorname{Re} h(z)-\left|\frac{g(z)}{g^{\prime}(z)} h^{\prime}(z)\right| \\
& \geqq \operatorname{Re} h(z)-|z|(1+|z|) \frac{2 \operatorname{Re} h(z)}{1-|z|^{2}} \\
& =\frac{1-3|z|}{1-|z|} \operatorname{Re} h(z) .
\end{aligned}
$$

Thus, for $|z|<\frac{1}{3} \operatorname{Re}\left\{f^{\prime}(z) / g^{\prime}(z)\right\}>0$. This shows that $f(z)$ is univalent and close-to-convex for $|z|<\frac{1}{3}[4]$.

Let us show that $f(z)$ maps $|z|<\frac{1}{3}$ onto a starlike domain.

$$
\begin{aligned}
\frac{z f^{\prime}(z)}{f(z)} & =\frac{z g^{\prime}(z)}{g(z)}+\frac{z h^{\prime}(z)}{h(z)} \\
\operatorname{Re}\left\{\frac{z f^{\prime}(z)}{f(z)}\right\} & \geqq \operatorname{Re}\left\{\frac{z g^{\prime}(z)}{g(z)}\right\}-\left|\frac{z h^{\prime}(z)}{h(z)}\right| \\
& \geqq \frac{1}{1+|z|}-\frac{2|z|}{1-|z|^{2}}=\frac{1-3|z|}{1-|z|^{2}} .
\end{aligned}
$$

For $|z|<\frac{1}{3} \operatorname{Re}\left\{z f^{\prime}(z) / f(z)\right\}>0$. Thus, $f(z)$ is starlike in $|z|<\frac{1}{3}$.

Theorem 4 gives the radius of univalence for the class of functions considered. In order to show this let $f(z)=\left(z+z^{2}\right) /(1-z)^{2}, g(z)$ 
$=z /(1-z)$. Then, $g(z)$ is univalent and convex for $|z|<1$. Here, $h(z)=(1+z) /(1-z)$ and therefore $\operatorname{Re} h(z)>0$. This function $f(z)$ is univalent in no circle $|z|<r$ with $r>\frac{1}{3}$ since $f^{\prime}\left(-\frac{1}{3}\right)=0$.

TheOREM 5. Suppose that $f(z)=z+a_{2} z^{2}+\cdots$ and $g(z)=z$ $+b_{2} z^{2}+\cdots$ are analytic for $|z|<1$ and $g(z)$ is univalent in $|z|<1$. If $\operatorname{Re}\{f(z) / g(z)\}>0$ for $|z|<1$ then $f(z)$ is univalent in $|z|<1 / 5$.

PROOF. Let $h(z)=f(z) / g(z)=1+c_{1} z+\cdots, \operatorname{Re} h(z)>0$ for $|z|<1$.

To show that $f(z)$ is univalent in $|z| \leqq r$ it suffices to show that $f(z)$ is univalent on $|z|=r$. Let $z_{1} \neq z_{2},\left|z_{1}\right|=\left|z_{2}\right|=r$. Then, $f\left(z_{1}\right)=f\left(z_{2}\right)$ can be written

$$
\frac{1}{g\left(z_{1}\right)} \frac{g\left(z_{2}\right)-g\left(z_{1}\right)}{z_{2}-z_{1}}=-\frac{1}{h\left(z_{2}\right)} \frac{h\left(z_{2}\right)-h\left(z_{1}\right)}{z_{2}-z_{1}} .
$$

Thus, if

$$
\left|\frac{g\left(z_{2}\right)-g\left(z_{1}\right)}{g\left(z_{1}\right)\left(z_{2}-z_{1}\right)}\right|>\left|\frac{h\left(z_{2}\right)-h\left(z_{1}\right)}{h\left(z_{2}\right)\left(z_{2}-z_{1}\right)}\right|
$$

then $f(z)$ is univalent in $|z| \leqq r$.

Let $k(z)=(1-h(z)) /(1+h(z)),|k(z)|<1$ for $|z|<1$ and $k(0)=0$. Therefore $\left|k^{\prime}(z)\right| \leqq 1$ for $|z| \leqq \sqrt{2}-1[2$, p. 19]. From the representation $k\left(z_{2}\right)-k\left(z_{1}\right)=\int_{z_{1}}^{z_{2}} k^{\prime}(z) d z$ where the path of integration is the line segment from $z_{1}$ to $z_{2}$ the estimate on $k^{\prime}(z)$ gives $\left|\left(k\left(z_{2}\right)-k\left(z_{1}\right)\right) /\left(z_{2}-z_{1}\right)\right|$ $\leqq 1$ for $r \leqq \sqrt{2}-1$. Expressing $h(z)$ in terms of $k(z)$ yields

$$
\begin{aligned}
\frac{h\left(z_{2}\right)-h\left(z_{1}\right)}{h\left(z_{2}\right)\left(z_{2}-z_{1}\right)} & =-2 \frac{k\left(z_{2}\right)-k\left(z_{1}\right)}{z_{2}-z_{1}} \frac{1}{\left(1+k\left(z_{1}\right)\right)\left(1-k\left(z_{2}\right)\right)} \\
\left|\frac{h\left(z_{2}\right)-h\left(z_{1}\right)}{h\left(z_{2}\right)\left(z_{2}-z_{1}\right)}\right| & \leqq \frac{2}{\left(1-\left|k\left(z_{1}\right)\right|\right)\left(1-\left|k\left(z_{2}\right)\right|\right)} \\
& \leqq \frac{2}{\left(1-\left|z_{1}\right|\right)\left(1-\left|z_{2}\right|\right)}=\frac{2}{(1-r)^{2}} .
\end{aligned}
$$

Here we have used Schwarz's lemma $|k(z)| \leqq|z|$.

Since $g(z)=z+b_{2} z^{2}+\cdots$ is analytic and univalent for $|z|<1$

$$
\left|\frac{g\left(z_{2}\right)-g\left(z_{1}\right)}{z_{2}-z_{1}}\right| \geqq\left|g\left(z_{1}\right) g\left(z_{2}\right)\right| \frac{1-r^{2}}{r^{2}}
$$

[3]. Using this estimate and the distortion theorem $|g(z)|$ $\geqq|z| /(1+|z|)^{2}$ we obtain 


$$
\left|\frac{g\left(z_{2}\right)-g\left(z_{1}\right)}{g\left(z_{1}\right)\left(z_{2}-z_{1}\right)}\right| \geqq \frac{1-r}{r(1+r)} .
$$

Therefore, $f(z)$ is univalent in $|z| \leqq r$ if $r \leqq \sqrt{2}-1$ and $(1-r) /(r(1+r))>2 /(1-r)^{2}$. The last inequality is equivalent to $1-5 r+r^{2}-r^{3}>0$. Since the equation $1-5 r+r^{2}-r^{3}=0$ has one positive root $r_{0}$, where $0.20<r_{0}<0.21$, we can infer that $f(z)$ is univalent in $|z|<r_{0}$. In particular, $f(z)$ is univalent in $|z|<1 / 5$.

The circle $|z|<r_{0}$ is not the circle of univalence for the functions $f(z)$ which satisfy the hypotheses of Theorem 5 . If it were then we must have $|g(z)|=|z| /(1+|z|)^{2}$ for some $z$. This estimate holds only for the functions $g(z)=z /(1+\epsilon z)^{2}$ where $|\epsilon|=1$. Since these functions are starlike for $|z|<1$ Theorem 3 implies that $f(z)$ would be univalent $|z|<2-\sqrt{3}$. However, $2-\sqrt{3}=0.267 \cdots>r_{0}$.

\section{REFERENCES}

1. J. W. Alexander, Functions which map the interior of the unit circle upon simple regions, Ann. of Math. 17 (1915), 12-22.

2. C. Carathéodory, Theory of functions, Vol. 2, Chelsea, New York, 1954.

3. G. M. Golusin, On distortion theorems and coefficients of univalent functions, Mat. Sb. (N. S.) 19 (61) (1946), 183-202. 185.

4. W. Kaplan, Close-to-convex schlicht functions, Michigan Math. J. 1 (1952), 169-

5. T. MacGregor, Functions whose derivative has a positive real part, Trans. Amer. Math. Soc. 104 (1962), 532-537.

6. A. Marx, Untersuchungen über schlichte Abbildungen, Math. Ann. 107 (1932), 40-67.

7. K. Noshiro, On the univalency of certain analytic functions, J. Fac. Sci. Hokkaido Univ. 2 (1934), 89-101.

8. G. Polya and G. Szegö, Aufgaben und Lehrsätze aus der Analysis, Vol. 1, Springer, Berlin, 1954.

9. E. Strohhäcker, Beiträge zur Theorie der schlichten Funktionen, Math. Z, 37 (1933), 356-380.

10. T. Umezawa, On the multivalency of analytic functions, J. Math. Soc. Japan 4 (1952), 279-285.

\section{Lafayette College}

